\title{
Localized states near the Abrikosov vortex core in type-II superconductors within zero-range potential model
}

\author{
V. L. Kulinskii, D. Yu. Panchenko \\ Department of Theoretical Physics, Odessa National University, Dvoryanskaya 2, 65082 \\ Odessa, Ukraine \\ kulinskij@onu.edu.ua,dpanchenko@onu.edu.ua
}

PACS 74.25.Jb, 74.25.Ha

DOI 10.17586/2220-8054-2015-6-3-353-360

\begin{abstract}
We propose to treat the lowest bound states near the Abrikosov vortex core in type-II superconductors on the basis of the self-adjoint extension of the Hamiltonian of Aharonov-Bohm type with the localized magnetic flux. It is shown that the Hamiltonian for the excitations near the vortex core can be treated in terms of the generalized zero-range potential method when the magnetic field penetration depth $\delta$ is much greater than the coherence length $\xi$ i.e. in the limit $\varkappa=\delta / \xi \gg 1$. In addition, it is shown that in this limit it is the singular behavior of $\mathrm{d} \Delta /\left.\mathrm{dr}\right|_{r=0}$ and not the details of the order parameter $\Delta(\mathbf{r})$ profile that is important. In support of the proposed model, we reproduce the spectrum of the Caroli-de Gennes-Matricon states and provide direct comparison with the numerical calculations of Hayashi, N. et al. [Phys. Rev. Lett. 80, p. 2921 (1998)]. In contrast to the empirical formula for the energy of the ground state in Hayashi, N. we use no fitting parameter. The parameters for the boundary conditions are determined in a self-consistent manner with Caroli-de Gennes-Matricon formula.
\end{abstract}

Keywords: Abrikosov vortex; bound states; zero-range potential.

Received: 18 February 2015

\section{Introduction}

Understanding the electronic structure of the vortex core in superconductors and the spectrum of excitations in its vicinity are important for the technology of the superconducting materials, as the low temperature limit of their spectrum is known to determine their static and dynamic properties. In the seminal work of [1], the existence of bounded states localized near the vortex core was predicted. A striking feature of the Caroli-de GennesMatricon (CdGM) solution is that the energy spectrum similar to the Landau levels with the effective region of localization is on the order of the vortex core radius $\xi_{1}$. This radius corresponds to the effective field of the order's upper critical limit $H_{c_{2}} \simeq \varkappa \gg 1$ [2]; that is, the field above which the superconductivity is destroyed. The appearance of the effective magnetic field of order $H_{c_{2}}$ is due to coherent Andreev reflection from the Cooper pair condensate which is spatially characterized by the spatially profile of the order parameter (the amplitude of the wave function of superconducting condensate) $\Delta(\mathbf{r})$ [3]. Also, the spectrum of the lowest bound states does not essentially depend on the specific spatial profile of the order parameter $\Delta(r)$. In fact, the linear dependence these states' energies on the angular momentum quantum number $\mu$ is determined by the limiting slope parameter $\mathrm{d} \Delta /\left.\mathrm{dr}\right|_{r=0}$. Self-consistent treatment in [4] showed that this slope parameter diverges in the quantum limit $T \rightarrow 0$. Shrinking of the core region leads to a reduction in the number of bound states $[5,6]$. The singular behavior of the order parameter is expected from the 
general reasonings about the gapless character of the fermionic excitations [7]. According to [7], the structure of $\Delta(\mathbf{r})$ can be even more complex and is characterized by additional scale $\xi_{1} \lesssim \xi_{B C S}=\xi_{0} / \pi$, where $\xi_{0}=v_{F} / \Delta_{\infty}{ }^{*}$, which separates the regions at the point where the jump of the derivative of the order parameter occurs. The quantity $\xi_{1}$ also determines the distance where the supercurrent density reaches its maximum $[8,9]$. So, we treat the distance $\xi_{1}$ as another characteristic length scale of the vortex core. Thus, the structure of the vortex core is far from trivial even in the limit $\varkappa \rightarrow \infty$ due to singularities caused by both the point-like structure of the defect and the spatial distribution of $\Delta(r)$. As a result, the electronic structure of the vortex core and the behavior of the order parameter are strongly correlated in the limit $T \rightarrow 0$ [8].

The aim of this paper is to propose a model Hamiltonian to describe the lowest bound state for conventional s-wave superconductors of the CdGM branch which is explicitly based on the singular behavior of the slope $\mathrm{d} \Delta(\mathbf{r}) /\left.\mathrm{dr}\right|_{r \rightarrow 0}$ in the quantum limit. The independence of the spectrum from the specific spatial profile of the order parameter $\Delta(\mathbf{r})$ follows directly. The concept is based on the results from [10,11], where the self-adjoint extensions for the Aharonov-Bohm (AB) Hamiltonian were studied. The key parameter is the fraction of the flux quantum $\Phi_{\text {core }}$ localized within the core. It should be noted that the standard $\mathrm{AB}$ effect for the Abrikosov vortex was considered for the scattering states and had little impact on the CdGM bound states [12]. From this point of view, the important result of [11] is that boundary conditions exist such that there is a bound state in the vicinity of the localized magnetic flux. This state is qualitatively different from the bound state in the potential well because it is caused in essence by the localized magnetic flux. This grounds the possibility of the treatment of the lowest bound states of the Bogolubov-de Gennes Hamiltonian with the help of self-adjoint extensions for AB Hamiltonian. Thus, we give physical interpretation for the nonstandard boundary conditions (or equivalently the self-adjoint extensions) for the AB Hamiltonian.

The structure of paper is as follows. In Section 1, we consider the relation between the BdG Hamiltonian for the quasiparticle excitations and the self-adjoint extension of the Aharonov-Bohm (AB) Hamiltonian. We show that these Hamiltonians are equivalent for the low lying energy states localized near the vortex core. In Section 2, we use the above result to study the dependence of the bound state energy on the relevant parameters and show how this can be used to explain the results of [5] in our approach. In the conclusion, the summary of the results is given and some problems for the further studies are listed.

\section{The Hamiltonian reduction for the low lying bound states}

Theoretical investigations of the quasiparticle spectrum around the vortex structure in the clean limit at low temperatures is based on the Bogoliubov-de Gennes Hamiltonian $(\mathrm{BdGH})[1]$ :

$$
\hat{H}=\sigma_{z}\left\{\left(\hat{\mathbf{p}}-\sigma_{z} \frac{e}{c} \mathbf{A}-\frac{1}{2} \sigma_{z} \nabla \theta\right)^{2}-E_{F}\right\}+\sigma_{x} \Delta(r)
$$

where $E_{F}$ is the Fermi energy, the vector potential $\mathbf{A}$ of the applied magnetic field of the order's lower critical field $H_{c_{1}}$, that is, the lowest field at which formation of vortices in a type-II superconductor becomes thermodynamically favorable while the gradient term is for the magnetic field localized in the vortex. The order parameter $\Delta(\mathbf{r})$ has obvious asymptotic

${ }^{*} \hbar=1, m=1 / 2$ in this work. 
behavior:

$$
\Delta(r)=\left\{\begin{array}{lll}
0 & \text { if } & r \rightarrow 0 \\
\Delta_{\infty} & \text { if } & r \rightarrow \infty
\end{array}\right.
$$

and should be determined consistently. To find the spectrum for (1), some specific model for $\Delta(r)$ can be used. Commonly, $\Delta(r)$ is taken in the form (see [12-14]):

$$
\Delta(r)=\Delta_{\infty} \tanh \frac{r}{\xi_{0}} .
$$

At the low temperature limit, because of Kramer-Pesch anomaly [15], the increase of $\Delta(r)$ to the asymptotic value $\Delta_{\infty}$ occurs at a distance $\xi_{1}$, much smaller than $\xi_{0}$, namely $\xi_{1} \simeq k_{F}^{-1}[16]:$

$$
\begin{gathered}
\Delta(r)=\Delta_{\infty} \frac{r}{\xi_{1}}+\ldots \\
\frac{E_{\mu}}{\Delta_{\infty}}=\left.\frac{2 \mu}{k_{F}} \frac{\mathrm{d} \Delta(\mathrm{r}) / \Delta_{\infty}}{\mathrm{dr}}\right|_{\text {Caroli et al. [1] }} ^{\frac{2 \mu}{k_{F} \xi_{0}}} \text { or } \frac{2 \mu}{k_{F} \xi_{1}} \\
\text { Kramer\&Pesch [4] } \\
\frac{2 \mu}{k_{F} \xi_{0}} \ln \left[\frac{\xi_{0}}{2 \xi_{1}}\right], \quad \xi_{1} \ll \xi_{0}
\end{gathered}
$$

due to presence of two length scales $\xi_{1}$ and $\xi_{0}$.

It is natural that for the materials with $\varkappa \gg 1$, the vortex can be considered as a point-like singularity due to the localized magnetic flux, similar to the situation of the AB Hamiltonian. As has been said above, the appearance of the bound state is due to reflection of the excitation from the Bose condensate of Cooper pairs which is equivalent to the effective magnetic field of the order $H_{c_{2}} \propto \varkappa$. Therefore we can interchange the $\Delta$-potential term in the Hamiltonian (1) by the field of the localized magnetic flux:

$$
\mathbf{A}(r)=\frac{\Phi_{\text {core }}}{2 \pi r} \mathbf{e}_{\theta} .
$$

The parameter $\alpha=\left\{\Phi_{\text {core }} / \Phi_{0}\right\}$ is the fraction of the magnetic flux corresponding to the region of the localization of the size $\xi_{1}$. Here the magnetic flux quantum $\Phi_{0}=2 \pi \mathrm{c} / e$ corresponds to the excitation with charge $e$. The total flux of the Abrikosov vortex is $\Phi_{0} / 2$ so that $\alpha<1 / 2$. Also we will use the dimensionless variables $\tilde{r}=r / \xi_{0}$. Thus, the Hamiltonian (1) can be reduced to a much simpler one:

$$
\hat{H}=\hat{H}_{\alpha}^{(A B)}-E_{F},
$$

where $\hat{H}_{\alpha}^{(A B)}$ is the self-adjoint extension for the AB Hamiltonian

$$
H_{A B}=\hat{\mathbf{p}}^{2}+\frac{(\alpha+1 / 2)^{2}}{r^{2}} .
$$

The divergence of the slope $\mathrm{d}\left(\Delta / \Delta_{\infty}\right) / \mathrm{dr}$ at $r \rightarrow 0$ can be treated correctly via the introduction of a dimensionless parameter $\xi_{1} \mathrm{~d}\left(\Delta / \Delta_{\infty}\right) /\left.\mathrm{dr}\right|_{r=0}$. The latter determines the 
energy of the bound states $[1,15]$. It can be shown that $2 \mu \Delta_{\infty} /\left(k_{F} \xi_{0}\right)$ is similar to the energy spectrum of the Landau levels. The effective region of localization is on the order of the vortex core radius $\xi_{1}$, which corresponds to the effective field of order $H_{c_{2}}$. Taking into consideration that $\Delta_{\infty}=v_{F} / \xi_{0}$ and $\xi_{G L} / \xi_{B C S}=\pi \xi_{G L} / \xi_{0} \approx 0.74$ [17], for $T / T_{c} \ll 1 /\left(k_{F} \xi_{0}\right)$ at $k_{F} \xi_{0}>1$ where $\xi_{G L}$ is the Ginzburg-Landau coherence length and is as follows:

$$
\xi_{G L}=\frac{1}{2 \pi} \sqrt{\frac{\Phi_{0}}{H_{e f f}}}=\frac{1}{\sqrt{\pi \omega_{H}}} \sqrt{\frac{H}{H_{e f f}}} \quad \text { with } \quad H_{e f f}=\frac{H_{c_{2}}}{\pi}, \quad \omega_{H}=\frac{2 e H}{c}
$$

and therefore

$$
E_{\mu}=2 \mu \frac{\Delta_{\infty}}{k_{F} \xi_{0}}=\frac{4 \mu}{\xi_{0}^{2}} \approx 2 \mu \frac{\omega_{H}}{\pi} \frac{H_{e f f}}{H} .
$$

Also considering that in the quantum limit the size of the vortex core $\xi_{1}<\xi_{0}$ [4], then the magnetic flux which is localized in the vortex core is defined as $\Phi_{\text {core }}=2 \pi \xi_{1}^{2} H_{\text {eff }} / \pi=$ $2 \xi_{1}^{2} H_{\text {eff }}$. This choice of $H=H_{\text {eff }} / \pi$ corresponds to the Landau levels from Eq. (10). It is easy to show that $\alpha$ has the form:

$$
\alpha=\frac{\Phi_{\text {core }}}{\Phi_{0}}=\frac{1}{2}\left(\frac{\xi_{1}}{\pi \xi_{G L}}\right)^{2} \simeq\left(\frac{\xi_{1}}{\xi_{0}}\right)^{2}
$$

Note that parameter $\alpha$ can be measured experimentally [8].

Thus, the inner structure of the vortex is encoded into the parameters of the proper boundary conditions for (8). The parameters of these conditions are related to the physical parameters of the limit $\xi_{1} \rightarrow 0$ such as $k_{F} \xi_{1}$ and $\xi_{1} / \xi_{0}$ (note that $\delta_{L} \gg \xi_{0}$ corresponds to $\varkappa \rightarrow \infty)$ when the slope of the order parameter $\Delta$ becomes singular.

It should be noted that the quadratic dependence $\alpha$ on $\xi_{1} / \xi_{0}$ is due to the assumption of effective magnetic field homogeneity within the core while taking the singular limit. In general, some scaling behavior can be expected $\alpha \propto\left(\xi_{1} / \xi_{0}\right)^{\nu}$. To estimate the value of $\alpha$ for real materials and to check the scaling dependence, we use the experimental data of [5] for superconductors in which $1<k_{F} \xi_{0}<16$. This way, it is possible to find the dependence of $\alpha$ on the ratio $\xi_{1} / \xi_{0}$. In the quantum limit, the value $\xi_{1} / \xi_{0}$ lies in the $0.1-0.7$ interval. Based on the experimental data of [5], we can plot on a logarithmic scale the dependence $\xi_{1} / \xi_{0}$ of $k_{F} \xi_{0}$ in the quantum limit for different values of $k_{F} \xi_{0}$. This dependence is shown in Fig. 1 and is approximated by the formula:

$$
\ln \xi_{1} / \xi_{0}=\ln 3 / 4-0.72 \ln k_{F} \xi_{0},
$$

which leads to:

$$
\alpha=\frac{1}{2(0.74)^{2}}\left(\frac{3}{4\left(k_{F} \xi_{0}\right)^{0.72}}\right)^{2} \approx \frac{1}{2} \frac{1}{\left(k_{F} \xi_{0}\right)^{1.44}}<\frac{1}{2} .
$$

For example, in the quantum limit for the $Y B C O$-superconductor $\left(k_{F} \xi_{0} \sim 4\right.$ with $T_{c}=90 K$ [18]) we get $\alpha \sim 0.1$. It is natural that for $k_{F} \xi_{0} \gg 1$ the parameter $\alpha$ vanishes. Now, we use the general results of [11] on spectrum of the model Hamiltonian of AB type (8).

\section{Bound states for the model Hamiltonian}

The nontrivial spectrum in extended AB problem is determined by the radial part of the AB Hamiltonian in the cylindrical coordinates:

$$
-\frac{\mathrm{d}^{2}}{\mathrm{~d} \tilde{\mathrm{r}}^{2}}-\frac{1}{\tilde{r}} \frac{\mathrm{d}}{\mathrm{d} \tilde{\mathrm{r}}}+(\alpha+\mu)^{2} \frac{1}{\tilde{r}^{2}}
$$




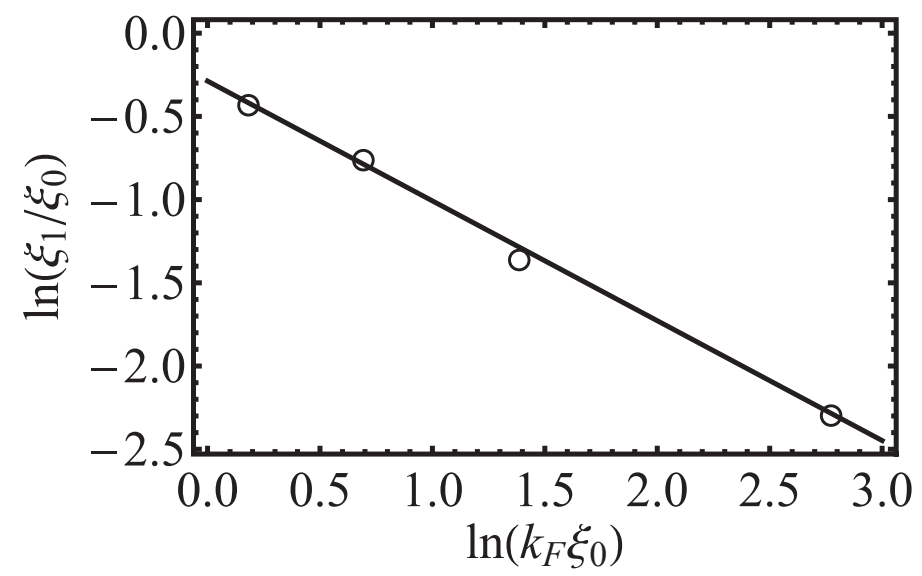

Fig. 1. The log-log plot for dependence $\xi_{1} / \xi_{0}$ on $k_{F} \xi_{0}$. The points represent the data from [5].

According to the theory of self-adjoint extensions, for the AB Hamiltonian, there is generally a 4-parameter set of boundary conditions for each $\alpha$ value [11]. But, if one requires that the Hamiltonian commutes with the angular momentum operator then this can be reduced to only 2 parameters [19]. In our case, $\mu=1 / 2$, there is only a one-parameter set of boundary conditions for each value of $\alpha$ :

$$
\Phi_{1}=b \Phi_{2},
$$

where

$$
\begin{gathered}
\Phi_{1}(\psi):=\lim _{r \rightarrow 0} r^{D} \int_{0}^{2 \pi} \psi(r, \theta) \mathrm{e}^{\mathrm{i} \theta / 2} \mathrm{~d} \theta / 2 \pi \\
\Phi_{2}(\psi):=\lim _{r \rightarrow 0} r^{-D}\left[\int_{0}^{2 \pi} \psi(r, \theta) \mathrm{e}^{\mathrm{i} \theta / 2} \mathrm{~d} \theta / 2 \pi-\mathrm{r}^{-\mathrm{D}} \Phi_{1}(\psi)\right] \\
D=1 / 2+\alpha
\end{gathered}
$$

and $b$ is the corresponding parameter of the boundary condition. The energy of the ground state is

$$
\frac{E_{0}(\alpha)}{\Delta_{\infty}}=\frac{2}{k_{F} \xi_{0}}\left(-\frac{b \Gamma(1 / 2-\alpha)}{\Gamma(3 / 2+\alpha)}\right)^{-2 /(1+2 \alpha)} .
$$

Note that both $\alpha$ and $\mu$ parameters enter the Hamiltonian (14) in the same way. We need to determine the slope of the dispersion law of (5) using (18) at $\Delta_{\infty}$ fixed. To do this, we take into account that the eigenvalues of (14) depend on the combination $\alpha+\mu$. Therefore if the gap $\Delta_{\infty}$ is fixed, then:

$$
\partial\left(E_{0}(\alpha)\right) /\left.\partial \alpha\right|_{\alpha=0}=-\partial\left(E_{\mu}\right) /\left.\partial \mu\right|_{\mu=0} .
$$

From Eq. (18) we obtain:

$$
\left.\frac{\partial E_{0}(\alpha) / \Delta_{\infty}}{\partial \alpha}\right|_{\alpha=0}=\frac{2\left(1-\gamma+\ln \left(-\frac{b}{2}\right)\right)}{k_{F} \xi_{0} b^{2}},
$$


but, according to (5):

$$
\left.\frac{\partial E_{\mu} / \Delta_{\infty}}{\partial \mu}\right|_{\mu=0}=\left.\frac{2}{k_{F}} \frac{\mathrm{d} \Delta(\mathrm{r}) / \Delta_{\infty}}{\mathrm{dr}}\right|_{r=0}=\frac{2}{k_{F} \xi_{B C S}}=\frac{2 \pi}{k_{F} \xi_{0}},
$$

where $\gamma$ is the Euler's constant and $\lim _{r \rightarrow 0} \Delta(r)=\Delta_{\infty} r / \xi_{B C S}$. This choice of $\xi=\xi_{B C S}$ corresponds to the Landau levels with magnetic field of $H_{\text {eff }}$ from Eq. (10). Comparing Eq. (20) and Eq. (21) we obtain the equation for the parameter $b$ :

$$
\frac{\left(\gamma-1-\ln \left(-\frac{b}{2}\right)\right)}{b^{2}}=\pi
$$

which has the solution:

$$
b=-\sqrt{\frac{W\left(8 \mathrm{e}^{2 \gamma-2} \pi\right)}{2 \pi}} \approx-0.53
$$

where $W$ is the Lambert $W$-function.

\subsection{Comparison with numerical calculations}

Self-consistent numerical solution of the Bogoliubov-de Gennes equations in the quantum limit for a clean $s$-wave superconductor was performed in [5]. There, it was shown that the shrinking of the core region leads to a reduction in the number of bound-quasiparticle state energy levels. For large $k_{F} \xi_{0}$ values, the energy of the ground state $(\mu=1 / 2)$ was fitted by the empirical expression (we use the notations of [5]):

$$
\frac{E_{1 / 2}}{\Delta_{\infty}}=\frac{\ln \left(k_{F} \xi_{0} / 0.3\right)}{2 k_{F} \xi_{0}}
$$

Now, using Eq. (13) and Eq. (23) it is possible to obtain the corresponding asymptotic for the from Eq. (18). For $k_{F} \xi_{0} \gg 1$, we get the following asymptotic behavior:

$$
\frac{E_{0}(\alpha)}{\Delta_{\infty}}=\frac{\pi}{k_{F} \xi_{0} W\left(8 \mathrm{e}^{2 \gamma-2} \pi\right)}+\alpha \frac{2 \pi}{k_{F} \xi_{0}}+O\left(\alpha^{2}\right) \underset{\alpha \rightarrow 0}{\approx} \frac{1.75}{k_{F} \xi_{0}} .
$$

Such behavior is due to the fact that $\alpha \rightarrow 0$ the energy $E_{0}(\alpha)$ tends to the CdGM ground

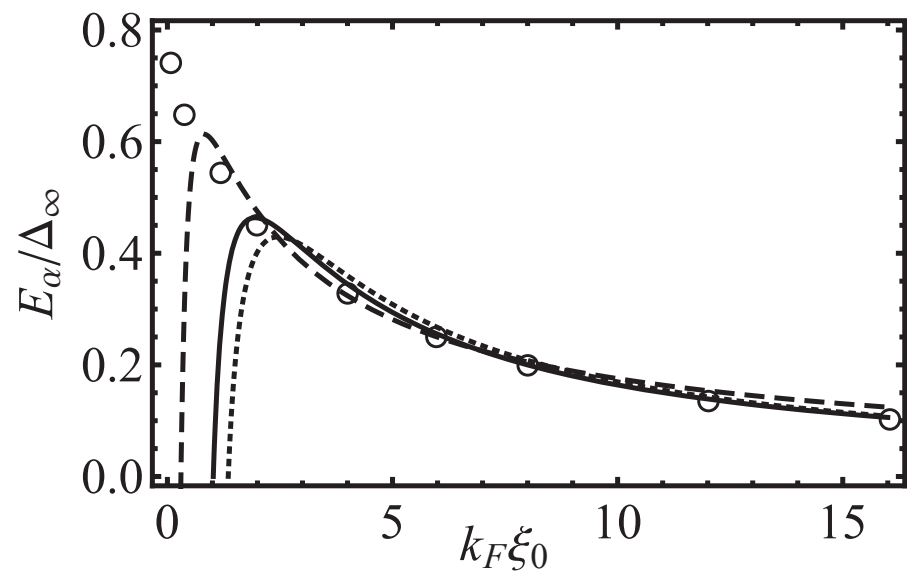

FIG. 2. The energy of ground state $E_{0} / \Delta_{\infty}$ as a function of $k_{F} \xi_{0}$. The solid curve shows the result of Eq. (18) at $b$ given by Eq. (23). The dashed line shows Eq. (24), the dotted line is the dependence of $E_{0}(\alpha) / \Delta_{\infty}$ on $k_{F} \xi_{0}$ for $\alpha \simeq\left(k_{F} \xi_{0}\right)^{-2}$. The open circles correspond to the numerical calculations of [5]. 
state. In Fig. 2 the comparison of our result with that of Hayashi N., et. al. [5] is shown. Note that the asymptotic behavior (25) of the ground state energy differs from the empirical result of Hayashi N., et. al. (24) and has better agreement for large values $k_{F} \xi_{0}$. Additionally, no fitting parameter was not used.

\section{Conclusion}

The main result of this paper is twofold. First, we have shown that the Kramer-Pesch anomaly for the order parameter slope $\mathrm{d} \Delta(\mathrm{r}) / \mathrm{d} \mathrm{r}$ in the core of the Abrikosov vortex can be described via non-standard boundary conditions for the Aaharonov-Bohm Hamiltonian. In such a manner, the well-known fact that CdGM spectrum is determined by the limiting slope of the order parameter $\mathrm{d} \Delta(\mathrm{r}) / \mathrm{d} \mathrm{r}$ acquires natural explanation. The analytical expression for the ground state energy $E_{0}$ as a function of the vortex core radius $\xi_{0}$ is obtained and shows good correspondence with known results derived from the numerical solution of BdG equations.

The second issue is that the non-standard boundary conditions for the AaharonovBohm Hamiltonian with the localized magnetic flux can be interpreted in physical terms as the Hamiltonian for the low-lying excitations near the Abrikosov vortex. This gives the possibility of using this approach for a more complicated situation, for example, a vortex pinned by the cylindrical defects [20].

\section{Acknowledgements}

The authors thank Prof. Vadim Adamyan for clarifying discussions. This work was partially supported by MES of Ukraine, Grant no. 0115U003208.

\section{References}

[1] Caroli C., de Gennes P.G., Matricon J. Bound Fermion states on a vortex line in a type II superconductor. Physics Letters, 1964, 9, 4, P. 307-309. http://dx.doi.org/10.1016/0031-9163(64)90375-0

[2] Bardeen J., Kümmel R., Jacobs A.E., Tewordt L. Structure of Vortex Lines in Pure Superconductors. Phys. Rev., 1969, 187(2), P. 556-569. http://dx.doi.org/10.1103/PhysRev.187.556

[3] Rainer D., Sauls J.A., Waxman D. Current carried by bound states of a superconducting vortex. Phys. Rev. B, 1996, 54(14), P. 10094-100106. http://dx.doi.org/10.1103/PhysRevB.54.10094

[4] Kramer L., Pesch W. Core structure and low-energy spectrum of isolated vortex lines in clean superconductors at $T \ll T_{c}$. Zeitschrift fur Physik A Hadrons and Nuclei, 1974, 269(1), P. 59-64. http://dx.doi.org/10.1007/BF01668869

[5] Hayashi N., Isoshima T., Ichioka M., Machida K. Low-Lying Quasiparticle Excitations around a Vortex Core in Quantum Limit. Phys. Rev. Lett., 1998, 80(13), P. 2921-2924. http://dx.doi.org/10.1103/PhysRevLett.80.2921

[6] Kato M., Maki K. Quasi-Particle Spectrum around a Single Vortex in Superconductors s-Wave Case. Progress of Theoretical Physics, 2000, 103(5), P. 867-879. http://dx.doi.org/10.1143/PTP.103.867

[7] Volovik G.E. Vortex Core Anomaly from the Gapless Fermions in the Core. Pis'ma Zh. Eksp. Teor. Fiz., 1993, 58(6), P. 455-460. http://www.jetpletters.ac.ru/ps/1189/article_17952.shtml

[8] Sonier J.E., Brewer J.H., Kiefl R.F. $\mu$ SR studies of the vortex state in type-II superconductors. Rev. Mod. Phys., 2000, 72(3), P. 769-811. http://dx.doi.org/10.1103/RevModPhys.72.769

[9] Sonier J.E. Investigations of the core structure of magnetic vortices in type-II superconductors using muon spin rotation. Journal of Physics: Condensed Matter, 2004, 16(40), P. S4499-S4513. http://dx.doi.org/10.1088/0953-8984/16/40/006

[10] Adami R., Teta A., On the Aharonov-Bohm Hamiltonian. Letters in Mathematical Physics, 1998, 43(1), P. 43-54. http://dx.doi.org/10.1023/A:1007330512611

[11] Dabrowski L., Štovíček P. Aharonov-Bohm effect with $\delta$-type interaction. Journal of Mathematical Physics, 1998, 39(1), P. 47-62. http://dx.doi.org/10.1063/1.532307 
[12] Cleary R.M. Scattering of Single-Particle Excitations by a Vortex in a Clean Type-II Superconductor. Phys. Rev., 1968, 175(2), P. 587-596. http://dx.doi.org/10.1103/PhysRev.175.587

[13] Shore J.D., Huang M., Dorsey A.T., Sethna J.P. Density of states in a vortex core and the zero-bias tunneling peak. Phys. Rev. Lett., 1989, 62(26), P. 3089-3092. http://dx.doi.org/10.1103/PhysRevLett.62.3089

[14] Gygi F., Schlüter M. Electronic tunneling into an isolated vortex in a clean type-II superconductor. Phys. Rev. B,1990, 41(1), P. 822-825. http://dx.doi.org/10.1103/PhysRevB.41.822

[15] Doettinger S.G., Huebener R.P., Kittelberger S. Kramer-Pesch effect and damping of the vortex motion in the cuprate superconductors. Phys. Rev. B, 1997, 55(9), P. 6044-6050. http://dx.doi.org/10.1103/PhysRevB.55.6044

[16] Gygi F., Schlüter M. Self-consistent electronic structure of a vortex line in a type-II superconductor. Phys. Rev. B, 1991, 43(10), P. 7609-7621. http://dx.doi.org/10.1103/PhysRevB.43.7609

[17] Schmidt V.V., The Physics of Superconductors: Introduction to Fundamentals and Applications, Springer, 1997. http://dx.doi.org/10.1007/978-3-662-03501-6

[18] Maggio-Aprile I., Renner C., Erb A., Walker E., Fischer O. Direct Vortex Lattice Imaging and Tunneling Spectroscopy of Flux Lines on $\mathrm{YBa}_{2} \mathrm{Cu}_{3} \mathrm{O}_{7-\delta}$. Phys. Rev. Lett., 1995, 75(14), P. 2754-2757. http://dx.doi.org/10.1103/PhysRevLett.75.2754

[19] Borg J.L., Pulé J.V. Pauli approximations to the self-adjoint extensions of the AharonovBohm Hamiltonian. Journal of Mathematical Physics, 2003, 44(10), P. 4385-4410. http://dx.doi.org/10.1063/1.1601298

[20] Mel'nikov A.S., Samokhvalov A.V., Zubarev M.N. Electronic structure of vortices pinned by columnar defects. Physical Review B (Condensed Matter and Materials Physics), 2009, 79(13), P. 134529/1-8. http://dx.doi.org/10.1103/PhysRevB.79.134529 